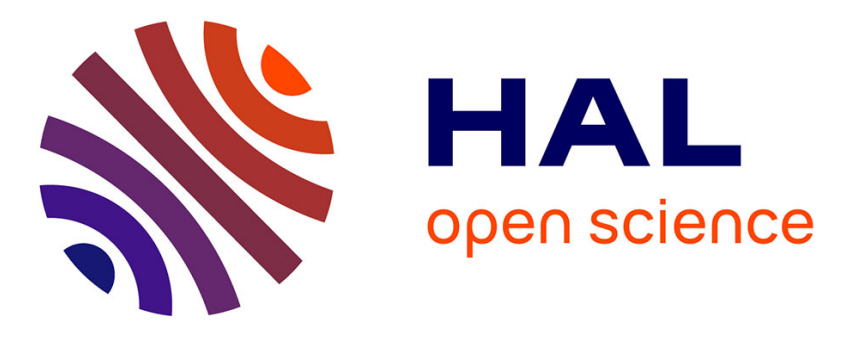

\title{
Blind Joint Polarization Demultiplexing and IQ Imbalance Compensation for M -QAM Coherent Optical Communications
}

Marwa Kazdoghli Lagha, Robin Gerzaguet, Laurent Bramerie, Mathilde Gay, Marie-Laure Chares, Christophe Peucheret, Pascal Scalart

\section{To cite this version:}

Marwa Kazdoghli Lagha, Robin Gerzaguet, Laurent Bramerie, Mathilde Gay, Marie-Laure Chares, et al.. Blind Joint Polarization Demultiplexing and IQ Imbalance Compensation for M -QAM Coherent Optical Communications. Journal of Lightwave Technology, 2020, 38 (16), pp.4213-4220. 10.1109/JLT.2020.2986601 . hal-02916203

\section{HAL Id: hal-02916203 \\ https://hal.science/hal-02916203}

Submitted on 17 Aug 2020

HAL is a multi-disciplinary open access archive for the deposit and dissemination of scientific research documents, whether they are published or not. The documents may come from teaching and research institutions in France or abroad, or from public or private research centers.
L'archive ouverte pluridisciplinaire HAL, est destinée au dépôt et à la diffusion de documents scientifiques de niveau recherche, publiés ou non, émanant des établissements d'enseignement et de recherche français ou étrangers, des laboratoires publics ou privés. 


\title{
Blind Joint Polarization Demultiplexing and IQ Imbalance Compensation for $M$-QAM Coherent Optical Communications
}

\author{
Marwa Kazdoghli Lagha, Robin Gerzaguet, Laurent Bramerie, \\ Mathilde Gay, Marie-Laure Chares, Christophe Peucheret and Pascal Scalart
}

\begin{abstract}
A novel joint polarization demultiplexing and IQ imbalance compensation scheme for coherent optical communications that uses global update is proposed and analyzed through numerical simulations. We describe the system model and derive its related equations. Next, we formulate our blind $M$-QAM arbitrary approach based on EASI algorithm and using the second order statistics of the observed signals. A comparison of the proposed joint method with the traditional CMA for polarization demultiplexing followed by BASS for IQ imbalance compensation is also reported. Evaluated metrics (EVM, MSE, BER) demonstrate its effectiveness compared with CMA cascaded with BASS algorithm.
\end{abstract}

Index Terms-Polarization demultiplexing, IQ imbalance compensation, Joint algorithm, CMA, MSE, EVM, BER, Optical communications

\section{INTRODUCTION}

$\mathbf{T}$ ODAY, with the explosive need of modern information networks, high bit rate fiber transmission has become essential. To accommodate this increase of data rates, digital signal processing (DSP) techniques are widely used in coherent optical communication systems with high-order modulation [1]. In fact, the introduction of high-order modulation formats, notably $M$-ary quadrature amplitude modulation ( $M$-QAM), enables to increase the bit rate while preserving the occupied bandwidth, at the expense of a higher required signal to noise ratio. The use of polarization-division multiplexing (PDM), combined with polarization and phasediversity coherent receivers, enables to further increase of the spectral efficiency. Indeed, PDM doubles the spectral efficiency by allocating two independent signals to two orthogonal polarizations tributaries [2]. At the receiver side, the two orthogonal polarizations of the incoming signal are projected onto two orthogonal polarization axes, leading to the subsequent detection of two complex fields.

Nevertheless, each of these received fields is actually a mixture of the two signals modulated onto each of the states of polarization at the transmitter. Hence, suitable techniques for polarization demultiplexing are required to ensure the recovery of the transmitted information. Different methods have been proposed to separate polarization-multiplexed signals tributaries, including the least mean square (LMS) algorithm with data training assistance [3] or blind solutions such as the constant modulus algorithm (CMA) [4]. Although these algorithms are very popular, they are modulation format dependent and they may have a relatively slow convergence rate [5].
Another system imperfection known as in-phase and quadrature (IQ) imbalance limits the system performance if not compensated in the DSP unit of the receiver [6]. IQ imbalance originates from the amplitude and phase mismatch between the in-phase and quadrature channels. It is introduced either at the transmitter side through incorrect electro-optical modulator settings or in the receiver front-end due to hardware hybrid circuit or trans-impedance amplifier imperfections [7]. Several works have proposed different methods to compensate IQ imbalance, among which the Gram-Schmidt orthogonalization procedure (GSOP) [8], the ellipse-correction (EC) approach [9] and the IQ imbalance compensation method based on the CMA [10]. The GSOP transforms non-orthogonal sets of samples to orthogonal ones based on statistical calculation while EC attempts to reshape the ellipse formed by received samples of the same nominal intensity level prior to carrier frequency offset compensation and carrier phase recovery, into a perfect circle based on simple transformations derived from the least square minimization approach. On the other hand, the CMA algorithm minimizes a cost function in order to estimate the IQ imbalance parameters. Nevertheless, all these techniques have some drawbacks. GSOP requires computing mathematical expectations of correlation coefficients, which when estimated using short-time statistics become sensitive to block size. Moreover, it needs an increased analog to digital converter resolution for high-order QAM formats [7]. Regarding EC compensation, it is not suited to high-order QAM formats nor useful at low optical signal-to-noise ratio (OSNR) values. The CMA method used for IQ imbalance compensation is shown in [10] not to be adapted to high-order modulation formats because it suffers from residual intersymbol interference and slow convergence. Furthermore, those three methods require a properly designed DSP unit entirely dedicated to IQ imbalance compensation.

Some contributions have proposed combined IQ compensation and polarization demultiplexing techniques [11, 12]. The problem is that these techniques use conventional two-bytwo butterfly-structured finite impulse response (FIR) filters, which increases the computational complexity. Additionally, they are modulation format dependent and suffer from a slow convergence rate when the modulation order of the received signal changes, as could happen in flexible optical network scenarios.

In this paper, we present a novel joint transmitter IQ imbalance (Tx-IQ imbalance) compensation and polarization- 
demultiplexing scheme. The proposed scheme is based on blind signal recovery using second order statistics for IQ imbalance compensation, known as blind adaptive source separation (BASS) algorithm [13] with a global updating of the equivariant adaptive separation via independence (EASI) algorithm for blind source separation [14].

The proposed algorithm is a useful tool for modulation format independent IQ imbalance compensation and polarization demultiplexing. Indeed, it is a blind approach that does not require known pilot or training signals, thus improving the spectral efficiency. Moreover, this algorithm shows good performance in terms of mean square error especially in highorder QAM modulation (up to $M=64$ ). Added to this, our proposed scheme is adaptive and suitable for agile transmission scenarios where modulation format or optical impairments experienced by the signal may vary over time and adaptable to probabilistic shaping modulation formats.

The rest of this paper is divided into five major sections. In section 2 , we present the system model and its formulation in the case of TX-IQ imbalance and PDM optical transmission. We detail, in section 3, the proposed scheme for joint Tx-IQ imbalance and polarization demultiplexing. Section 4 is dedicated to computer simulations demonstrating the benefits of our method, while section 5 reports a hardware complexity analysis. Finally, we conclude our paper in section 6 .

\section{SySTEM MODEL FOR TX-IQ IMBALANCE WITH PMD}

Figure 1 presents the simplified setup of a dual-polarization $M$-QAM coherent system in which the optical signal is subjected to fiber linear propagation effects as well as amplified spontaneous emission (ASE) noise.

First, $M$-QAM mapped data is generated from pseudo random binary sequences (PRBSs). The PRBSs are with lengths of $2^{11}-1$ and are used for generating the signal along polarization $\mathrm{X}$ and $\mathrm{Y}$, respectively. This is done by shifting by half the PRBS sequence for decorrelation between $\mathrm{X}$ and $\mathrm{Y}$ polarizations. These modulated data are then upsampled by 8 and pulse-shaped using a square-root raised cosine filter with a roll off of 0.5. A delayed version of the obtained signal represents the second polarization. In a second step, the four obtained digital streams are converted into analog signals thanks to four digital-to-analog converters. Next, the generated signals are used as tributaries in a polarization division multiplexing system. Here, light from a continuous wave $(\mathrm{CW})$ laser is split into two equal power contributions by a polarization beam splitter (PBS), modulated independently by two in-phase/quadrature modulators and then combined back in two orthogonal polarizations thanks to a polarization beam combiner (PBC).

Nonlinear distortions induced through transmission over the optical fiber are disregarded in the present studies. The considered transmission impairments include chromatic dispersion (CD) and polarization effects, namely polarization-mode dispersion (PMD). PMD is modelled as polarization rotation represented by a unitary matrix and differential group delay (DGD)between the orthogonal polarization tributaries. ASE from erbium-doped fiber amplifiers is modelled as additive white Gaussian noise (AWGN) for the optical field.
In order to convert the optical field into the electrical domain a coherent receiver is used. This latter contains a pair of $90^{\circ}$ optical hybrid circuits, one for each polarization, and two beam splitters. Next, the signal goes through photodetection, amplification and analog-to-digital conversion, and input to the entrance of the DSP stage.

Next, the models of the transmission impairments are detailed, including those originating from the linear channel as well as IQ imbalance at the transmitter side. In the following, it is assumed that the signals at each polarization are modeled as complex-valued random signals with zero mean and unit variance. $I_{n}$ is a square $n \times n$ identity matrix. Complex-conjugation is denoted by $(.)^{*}$. If $Z(t)=\left[z_{X}(t), z_{Y}(t)\right]^{T}$, where $z_{X}$ and $z_{Y}$ are the signals in both $X$ and $Y$ polarization and [.] $]^{T}$ is the transpose of the vector, then $\underline{\mathbf{Z}}(t) \stackrel{\text { def }}{=}\left[z_{X}(t), z_{X}{ }^{*}(t), z_{Y}(t), z_{Y}{ }^{*}(t)\right]^{T}$. Also, $[.]^{H}$ is the trans-conjugate of a vector or of a matrix.

Figure 2 shows the model of the dual-polarization transmission system with polarization and phase diversity coherent detection. Let $s_{p}(t)$ be the source signals in both polarizations, i.e. for $p=X$ or $p=Y$. $I_{s_{p}}(t)$ and $Q_{s_{p}}(t)$ are their in-phase and quadrature components, respectively. In the presence of IQ phase and amplitude imbalance at the transmitter, the complex representation of a continuous-wave optical signal at angular frequency $\omega$ on the $p$-polarization can be expressed as [15]:

$$
\begin{aligned}
m_{T x, p}(t) & =k_{1, p} e^{-j \omega t}+k_{2, p} e^{j \omega t}, \\
k_{1, p} & =\cos \left(\phi_{p}\right)+j \epsilon_{p} \sin \left(\phi_{p}\right), \\
k_{2, p} & =\epsilon_{p} \cos \left(\phi_{p}\right)+j \sin \left(\phi_{p}\right) .
\end{aligned}
$$

The parameters $\epsilon_{p}, \phi_{p}$ represent the amplitude and phase imbalance induced at the transmitter side for the signal in the $p$-polarization. Based on (1), the signal modulated on each polarization can, in the presence of IQ phase and amplitude imbalance, be expressed as

$$
u_{p}(t)=k_{1, p} s_{p}(t)+k_{2, p} s_{p}^{*}(t) .
$$

The distorted signals on each polarization are then multiplexed and transmitted over the optical fiber. The optical fiber linear impairments are modeled by

$$
\mathbf{H}(\omega)=\mathbf{J D}(\omega) \mathbf{C}(z, \omega),
$$

where:

$$
\mathbf{J}=\left(\begin{array}{cc}
\cos \alpha & e^{-j \theta} \sin \alpha \\
-e^{j \theta} \sin \alpha & \cos \alpha
\end{array}\right)
$$

is a Jones matrix representation of the random polarization rotation with random phase shifts between the two axes [16]. $\alpha$ and $\theta$ are azimuth and elevation rotation angles, respectively, that can make the signal state of polarization sweep over the entire Poincare sphere.

$$
\mathbf{D}(\omega)=\left(\begin{array}{cc}
e^{j \omega \tau / 2} & 0 \\
0 & e^{-j \omega \tau / 2}
\end{array}\right)
$$

represents the PMD-induced differential group delay $\tau$ between both polarization waves whose typical values range 


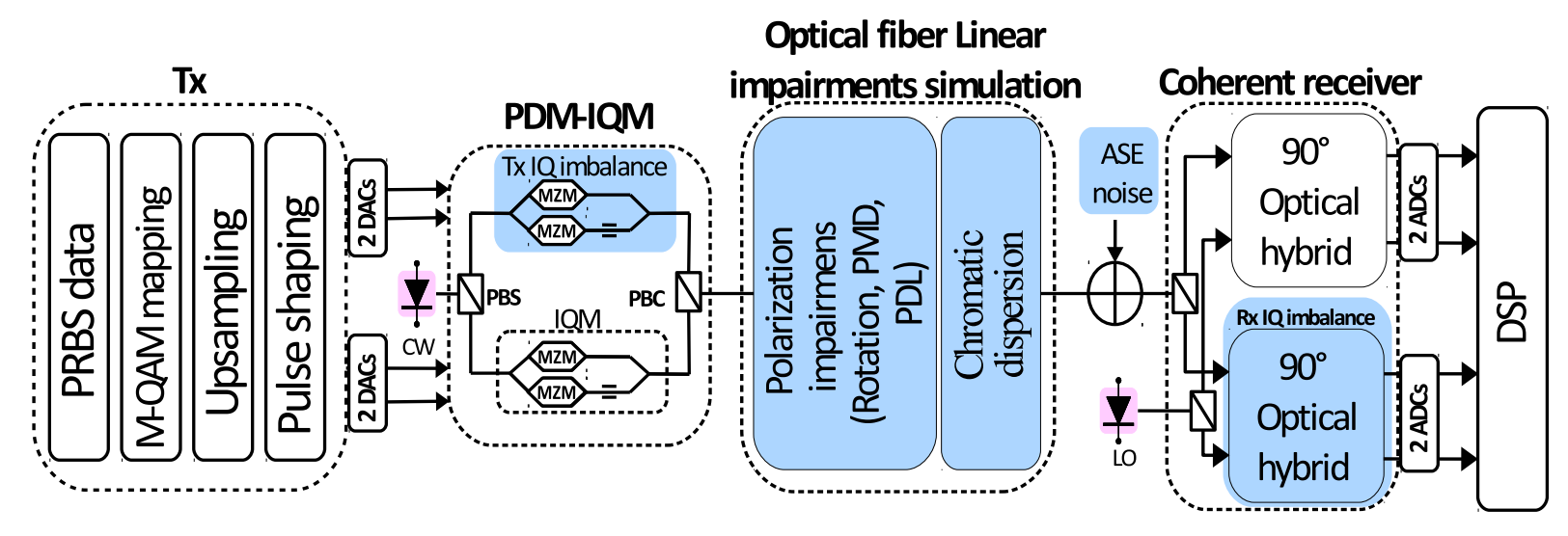

Figure 1: Simplified setup of dual-polarization $M$-QAM coherent system with linear impairments and noise.

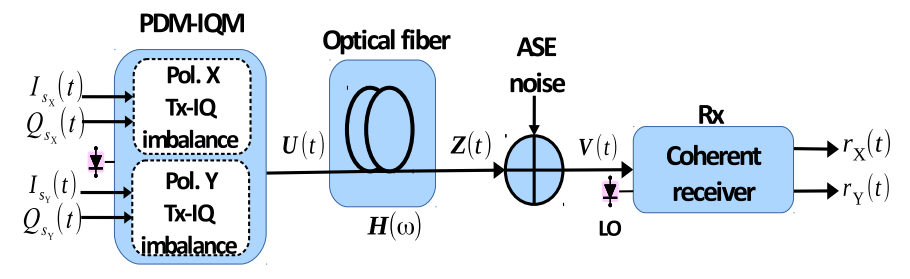

Figure 2: Model of optical transmission circuit.

between 1 and $100 \mathrm{ps}$ [17].

$\mathbf{C}(z, \omega)=e^{-\frac{j \lambda^{2} D z}{4 \pi c} \omega^{2}}$ corresponds to the frequency response of chromatic dispersion where $\lambda$ is the central wavelength of the transmitted optical wave, $D$ is the fiber chromatic dispersion coefficient, $z$ is the propagation distance and $c$ is the speed of light in vacuum [18].

In our case, we will study the optical fiber channel in the absence of polarization dependent loss (PDL). Numerical simulations demonstrate the robustness of the algorithm against PDL, but this point is outside the scope of our paper. Additionally, dynamic polarization multiplexing scenario was tested under numerical simulations and has proved the adaptability of the algorithm to this transmission conditions. Due to space constraints, we omit to show the numerical results in this letter. Moreover, fiber non-linearities will be neglected and suppose that chromatic dispersion is perfectly compensated by a digital filter with a fixed number of taps in the DSP unit. Differential group delay is also compensated. With these assumptions, the optical fiber model will be limited to the polarization rotator operation i.e. $\mathbf{H}=\mathbf{J}=\left(\begin{array}{ll}h_{1} & h_{2} \\ h_{3} & h_{4}\end{array}\right)$.

Next, the expression of the signals $\mathbf{V}(t)$ corrupted by ASE are derived

$$
\mathbf{V}(t)=\mathbf{J U}(t)+\mathbf{N}(t),
$$

with $\mathbf{N}(t)=\left[n_{X}(t), n_{Y}(t)\right]^{T}$, where $n_{X}(t)$ and $n_{Y}(t)$ are two independent complex circular white zero-mean Gaussian random variables with variance $N_{0} / 2$ per real dimension. $N_{0}$ is the power spectral density (per polarization) of ASE noise introduced by all the optical amplifiers along the link. We can derive from equations (2) and (6), the expression of the distorted signals related to the source signals in the form

$$
\underline{\mathbf{V}}(t)=\mathbf{G} \underline{\mathbf{S}}(t)+\underline{\mathbf{N}}(t) .
$$

In fact, as according to (2) the effect of IQ imbalance can be represented as a linear combination of the signal and its conjugate, it is worthy to represent the global model as a $4 \times 1$ vector taking into account the signal in both $X$ and $Y$ polarizations (for polarization mixing purpose) and their complex conjugates. Here, the $\mathbf{G}$ matrix englobes the effects of both Tx-IQ imbalances and the polarization rotation and is expressed by

$$
\mathbf{G}=\left(\begin{array}{cccc}
h_{1} k_{1, X} & h_{1} k_{2, X} & h_{2} k_{1, Y} & h_{2} k_{2, Y} \\
h_{1}^{*} k_{2, X}^{*} & h_{1}^{*} k_{1, X}^{*} & h_{2}^{*} k_{2, Y}^{*} & h_{2}^{*} k_{1, Y}^{*} \\
h_{3} k_{1, X} & h_{3} k_{2, X} & h_{4} k_{1, Y} & h_{4} k_{2, Y} \\
h_{3}^{*} k_{2, X}^{*} & h_{3}^{*} k_{1, X}^{*} & h_{4}^{*} k_{2, Y}^{*} & h_{4}^{*} k_{1, Y}^{*}
\end{array}\right) .
$$

In the next section, we will focus on the proposed algorithm enabling a blind procedure for recovering the original signals.

\section{JOINT ALGORITHM FOR TX-IQ IMBALANCE COMPENSATION AND POLARIZATION DEMULTIPLEXING}

\section{A. Formulation of the proposed algorithm}

Considering equation (7), our goal is to find the ideal compensation matrix $\mathbf{A}(t)$ that converges to the inverse of $\mathbf{G}$ in an adaptive manner (see figure 3). Hence, the demultiplexed and compensated signals $\underline{\tilde{\mathbf{S}}}(t)$ are given by:

$$
\underline{\tilde{\mathbf{S}}}(t)=\mathbf{A}(t) \mathbf{G} \underline{\mathbf{S}}(t)+\mathbf{A}(t) \underline{\mathbf{N}}(t) \stackrel{t \rightarrow \infty}{\longrightarrow} \underline{\mathbf{S}}(t)+\mathbf{A} \underline{\mathbf{N}}(t),
$$

where $\mathbf{A}(t) \stackrel{t \rightarrow \infty}{\longrightarrow} \mathbf{A}$.

In this step, both blind signal estimation for IQ imbalance compensation and the EASI algorithms are considered. The idea behind blind signal estimation for IQ imbalance (or BASS algorithm) is to whiten the received signals so that the complex conjugate component introduced by the Tx-IQ imbalance is eliminated. This algorithm requires circular or proper complex signals and uses second-order statistics for decorrelation [13]. Practically, this whitening problem is solved by using the principle of the natural gradient that leads to an adaptive rule converging to the first-order approximation of the inverse 


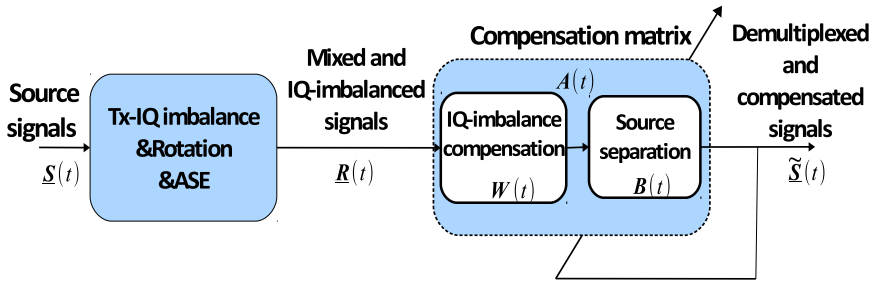

Figure 3: Adaptive and blind procedure for recovering the signals.

square root of the input covariance matrix. The adaptive rule is given by

$$
\mathbf{W}(t+\Delta t)=\mathbf{W}(t)-\mu\left[\underline{\mathbf{S}}_{W}(t) \underline{\mathbf{S}}_{W}(t)^{H}-I_{4}\right] \mathbf{W}(t),
$$

where $\mathbf{W}(t)$ is the whitening $4 \times 4$ matrix, $\mu$ is the adaptation step size and $\underline{\mathbf{S}}_{W}(t)=\mathbf{W}(t) \underline{\mathbf{R}}(t)$ are the adjusted signals.

At this stage IQ imbalance compensation is performed. However, the signals are kept polarization-multiplexed and another blind source separation algorithm needs to be introduced. Hence, we employ the EASI method for demultiplexing the mixed signals.

EASI is a blind source separation algorithm that requires some assumptions, namely statistical conditions on the input signals. In fact, this method involves a mutually statistically independent, zero mean and unit variance target signals, which is the case of our context of work. Also, the unknown mixing matrix (which, in our case, is the $\mathbf{H}$ matrix) must be full rank [14]. In the absence of IQ imbalance compensation, $\mathbf{R}(t)$ are the received signals, and $\tilde{\mathbf{S}}(t)$ is the EASI algorithm output, then the separating matrix update equation from the normalized EASI algorithm has the form

$$
\begin{aligned}
\mathbf{B}(t+\Delta t) & =\mathbf{B}(t)-\mu\left[\frac{\tilde{\mathbf{S}}(t) \tilde{\mathbf{S}}(t)^{H}-I_{2}}{1+\mu \tilde{\mathbf{S}}(t)^{H} \tilde{\mathbf{S}}(t)}\right. \\
& \left.+\frac{g(\tilde{\mathbf{S}}(t)) \tilde{\mathbf{S}}(t)^{H}-\tilde{\mathbf{S}}(t) g(\tilde{\mathbf{S}}(t))^{H}}{1+\mu\left|\tilde{\mathbf{S}}(t)^{H} g(\tilde{\mathbf{S}}(t))\right|}\right] \mathbf{B}(t),
\end{aligned}
$$

and $\tilde{\mathbf{S}}(t)=\mathbf{B}(t) \mathbf{R}(t)$, where $\mu$ is the adaptation step size, $\mathbf{B}(t)$ is the $2 \times 2$ separation matrix and $g($.$) is a component-$ wise nonlinear odd function. In this case, signal sources are successfully separated but the IQ imbalance persists. To combine both IQ compensation and demultiplexing procedures, a one stage solution is proposed. In the light of (10) and (11), the global updating rule is deduced. Instead of just looking to the signals in $\mathbf{R}(t)$, we take into consideration its complex conjugate components and we replace it by $\underline{\mathbf{R}}(t)$. In this case, the first term $\left(\underline{\tilde{\mathbf{S}}}(t) \underline{\tilde{\mathbf{S}}}(t)^{H}-I_{4}\right)$ accomplishes blind signal estimation for IQ imbalance compensation and the second term $\left(g(\underline{\tilde{\mathbf{S}}}(t)) \underline{\tilde{\mathbf{S}}}(t)^{H}-\underline{\tilde{\mathbf{S}}}(t) g(\underline{\tilde{\mathbf{S}}}(t))^{H}\right)$ implements the demultiplexing operation. Therefore, the proposed updating equation for both Tx-IQ imbalance compensation and polarization demultiplexing is given by

$$
\begin{array}{r}
\mathbf{A}(t+\Delta t)=\mathbf{A}(t)-\mu\left[\frac{\tilde{\tilde{\mathbf{S}}}(t) \underline{\tilde{\mathbf{S}}}(t)^{H}-I_{4}}{1+\mu \underline{\tilde{\mathbf{S}}}(t)^{H} \underline{\tilde{\mathbf{S}}}(t)}+\right. \\
\left.\frac{g(\underline{\tilde{\mathbf{S}}}(t)) \underline{\tilde{\mathbf{S}}}(t)^{H}-\underline{\tilde{\mathbf{S}}}(t) g(\underline{\tilde{\mathbf{S}}}(t))^{H}}{1+\mu\left|\underline{\tilde{\mathbf{S}}}(t)^{H} g(\underline{\tilde{\mathbf{S}}}(t))\right|}\right] \mathbf{A}(t) .
\end{array}
$$

\section{B. Overcoming the singularity problem}

Generally, algorithms of dynamic equalization realizing polarization demultiplexing suppose an independent convergence of their two outputs. In such a situation, a singularity problem could appear, i.e. each output converges to the same transmitted signal, preventing successful polarization demultiplexing. To deal with this issue, some methods using a cross correlation term of the cost function of the equalizer [19] or relying on the selection of a proper initial tap [18] have been proposed. In our compensation algorithm, we have dealt with the solution proposed in [18].

This procedure determines initial tap values so that degeneration of the two tributaries is prevented. Indeed, when considering only the polarization rotation impairments, the channel transfer matrix of the fiber is given by a unitary matrix

$$
\mathbf{G}=\left(\begin{array}{cccc}
h_{1} & 0 & h_{2} & 0 \\
0 & h_{1}^{*} & 0 & h_{2}^{*} \\
h_{3} & 0 & h_{4} & 0 \\
0 & h_{3}^{*} & 0 & h_{4}^{*}
\end{array}\right) .
$$

The optimal equalizer is given by the inverse of the $\mathbf{G}$ matrix as follows

$$
\mathbf{G}^{-1}=\left(\begin{array}{cccc}
v_{1} & 0 & v_{2} & 0 \\
0 & v_{1}^{*} & 0 & v_{2}^{*} \\
v_{3} & 0 & v_{4} & 0 \\
0 & v_{3}^{*} & 0 & v_{4}^{*}
\end{array}\right),
$$

where $v_{1}, v_{2}, v_{3}, v_{4}$, are expressed from $h_{1}, h_{2}, h_{3}, h_{4}$.

This matrix has a particular structure and could be exploited for the initialization of its coefficients. In this case, the singularity problem can be resolved. This additional constraint, which is added in the phase of initial convergence, is released after a certain time. In fact, in a second step, the compensation update for the two polarization is performed independently.

\section{Simulation RESUlts AND Discussions}

In this section, we evaluate the performance of our proposed joint Tx-IQ imbalance compensation and polarization demultiplexing algorithm, hereafter called, compared with the conventional CMA equalizer for polarization demultiplexing cascaded with the blind signal estimation for IQ compensation algorithm, hereafter labeled "CMA+BASS". The reason to work with the BASS algorithm rather than GSOP is based on earlier results [12], where it has been shown that BASS is less computationally complex and performs better in terms of error vector magnitude (EVM) and bit error rate (BER) than GSOP.

As shown in figure 4 , several metrics were calculated to compare the two aforementioned methods. 


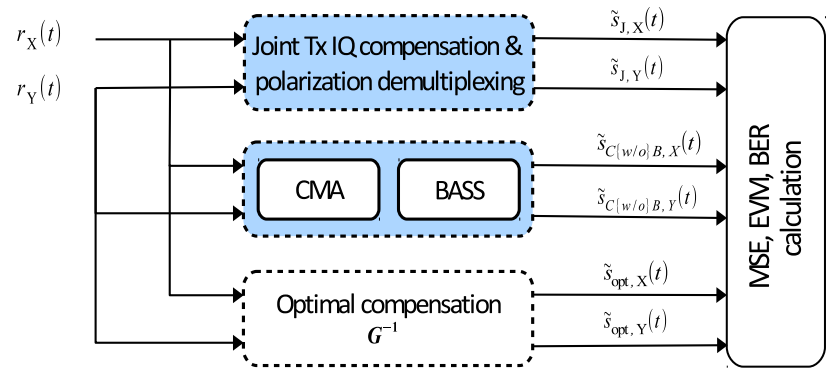

Figure 4: Presented methods and the studied comparative metrics.

Initially, the mean square error (MSE) is calculated. Here, the MSE is obtained by comparing the reference signal, which is obtained by optimal compensation $\tilde{s}_{\text {opt }, p}$, with the signals compensated by either the "joint" algorithm $\tilde{s}_{J, p}$ or by "CMA (with/without) BASS" $\tilde{s}_{C(w / o) B, p}$.

Figure 5 represents MSE curves obtained when the aforementioned adaptive algorithms are employed for 16-QAM modulation in three different use cases: (a) absence of Tx-IQ imbalance, (b) presence of Tx-IQ imbalance without "BASS" algorithm, and (c) presence of Tx-IQ imbalance with "BASS" algorithm. For all numerical simulations, the signal-to-noise ratio (SNR) is defined as the $E_{s} / N_{0}$ (energy per symbol to noise power spectral density ratio). In all cases, the SNR is fixed in simulations to $15 \mathrm{~dB}$, the chosen operating point for polarization rotation is set to $\left(\alpha=30^{\circ}, \theta=50^{\circ}\right)$, and the Tx-IQ imbalances parameters are $\left(\epsilon_{p}=0.1, \phi_{p}=6^{\circ}\right)$. The MSE calculation is averaged over 500 simulation runs and over the two polarizations. Also, it is worth mentioning that all step sizes are constant and fixed in a way that initial convergence speed is the same for all algorithms. Algorithms are therefore compared only by exploring the steady state convergence. In fact, for a specific application, the choice of one adaptive filter or algorithm over another could be determined by one or more of these factors: rate of convergence, misadjustment of the steady state, computational requirements and numerical robustness. The computational requirements and the numerical robustness are related to a practical issue. Regarding numerical simulations, by setting the algorithms to the same rate of convergence, we can compare their performance regarding the steady state misadjustment.

Figure 5a shows that when there is no IQ imbalance, the "joint" algorithm has a slightly poorer steady-state performance, i.e. the "joint" algorithm converges to a higher MSE value than CMA. This can be explained by the additional work of IQ imbalance compensation that has been done by the "joint" algorithm. When adding Tx-IQ imbalance, and without its compensation by the BASS algorithm, Fig. 5b depicts a huge steady-state performance difference between the two methods. In fact, the "joint" algorithm maintains the same steady-state level as without Tx-IQ imbalance, whereas the CMA algorithm proves to be non-operational for IQ compensation. But, by appending the BASS algorithm (Fig. 5c), the two-cascaded algorithms "CMA+BASS" can reach the same level of performance as the "joint" algorithm.

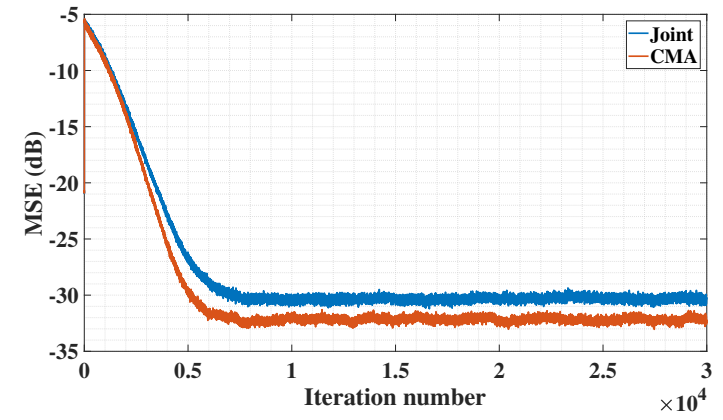

(a)

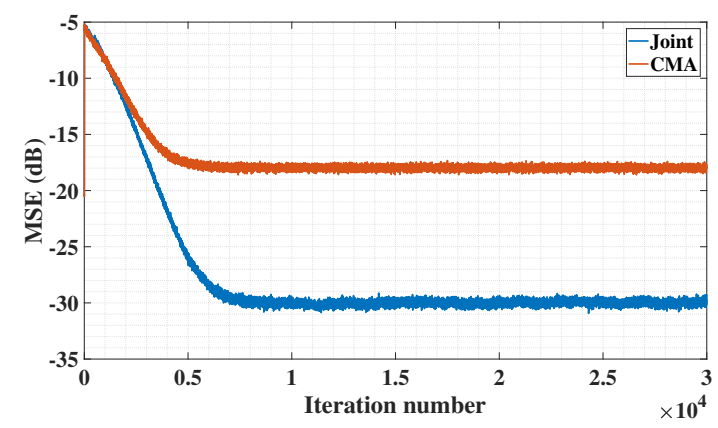

(b)

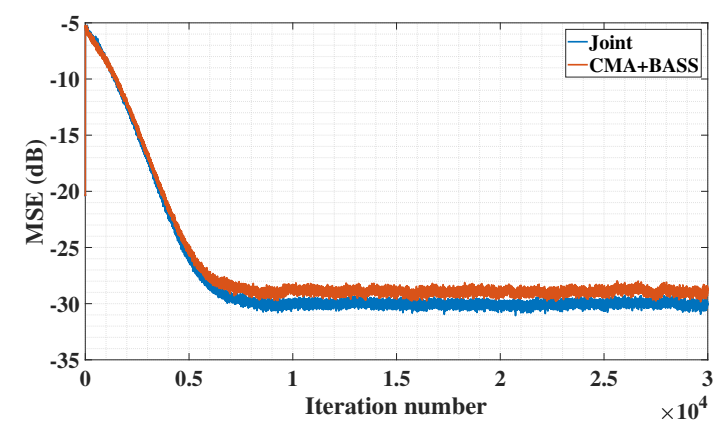

(c)

Figure 5: MSE curves (a) Without Tx-IQ imbalance, (b) With Tx-IQ imbalance and without BASS compensation, (c) With Tx-IQ and BASS compensation.

Next, we evaluate in Fig. 6 the average EVM surfaces (over $X$ and $Y$ polarizations) as a function of IQ gain imbalance (Fig. 6a) and IQ phase imbalance (Fig. 6b) for 16-QAM modulation in the presence of the same aforementioned polarization rotation parameters $\left(\alpha=30^{\circ}, \theta=50^{\circ}\right)$ and $15 \mathrm{~dB}$ SNR.

It is worthy to mention that the performance is evaluated in the steady-state regime (the asymptotic convergence part in the MSE sense). As can be seen, both algorithms achievements are degraded in the presence of any of the impairments (either the IQ-gain or the IQ-phase imbalance). Additionally, the phase imbalance is a more limiting effect than the gain imbalance. However, in all cases, the "joint" algorithm maintains better performance compared with "CMA+BASS". Indeed, the "joint" algorithm results in $0.2 \%$ better EVM values than the "CMA+BASS" at $10^{\circ}$ phase imbalance, whereas the results of the two algorithms are globally similar when gain imbalance is introduced. Fig. 7 depicts simulated performance of the 


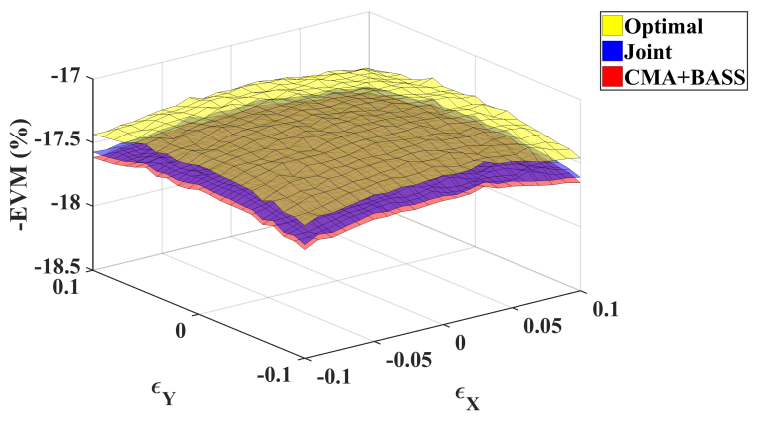

(a)

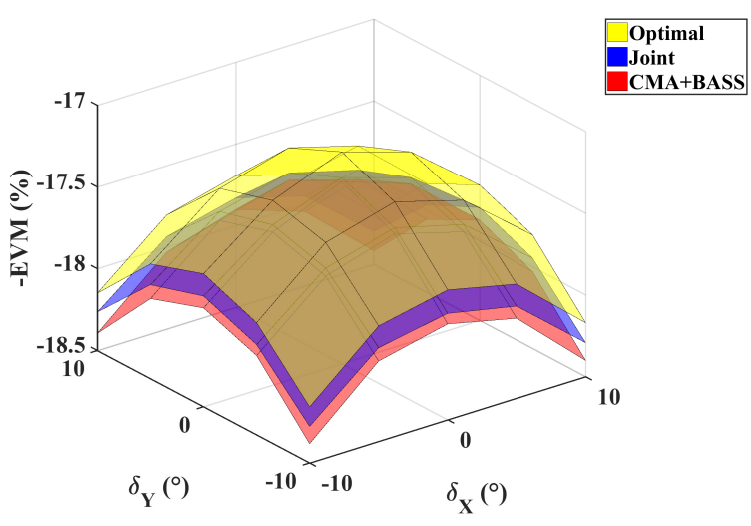

(b)

Figure 6: Average EVM surfaces as a function of a) the gain imbalance, b) phase imbalance.

presented algorithms in terms of EVM when varying the polarization rotation parameters $(\alpha$ and $\theta)$, in the presence of 0.1 gain imbalance and $6^{\circ}$ phase imbalance. The results are still averaged over $X$ and $Y$ polarizations and the introduced SNR is $15 \mathrm{~dB}$. We show that the "joint" algorithm outperforms cascaded "CMA+BASS" regardless of the introduced polarization rotation parameters.

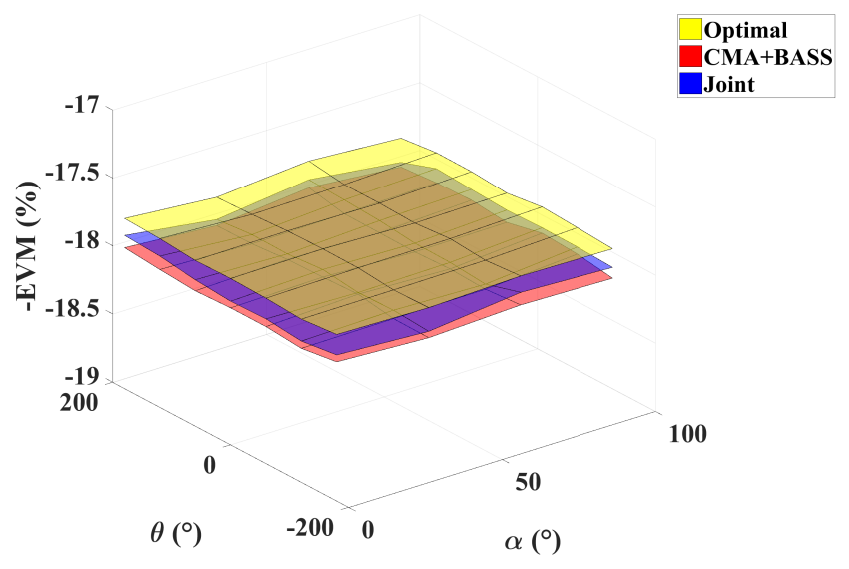

Figure 7: Average EVM surfaces as a function of the polarization rotation parameters with 16-QAM modulation.

We have investigated the BER evolution as a function of the SNR for 16-QAM signals in the presence of both Tx-IQ imbalance and polarization rotation impairments. We fix $\alpha=30^{\circ}, \theta=50^{\circ}, \epsilon_{p}=0.1, \phi_{p}=6^{\circ}$. Three BER curves are compared: the first is when the compensation is made by optimal matrix multiplication, the second is when the "CMA+BASS" method is used, and the third is when the "joint" algorithm is employed.

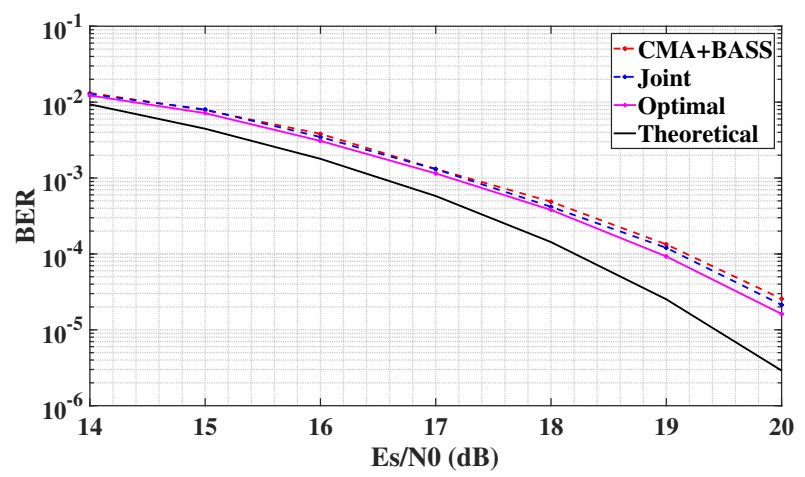

Figure 8: Evolution of the BER of 16-QAM signal as a function of the SNR in the presence of Tx-IQ imbalance and polarization rotation.

As can be seen in Fig 8, the "joint" method presents roughly the same performance as the CMA cascaded with the BASS algorithm in terms of BER metric. The distortion in EVM of the "CMA+BASS" comparing the "joint" did not have much impact on the algorithm in terms of BER, but this could be problematic for higher order modulation formats.

After having explored the "joint" algorithm performance under different impairments parameters and introduced SNRs, we will investigate its blind property to handle different modulation formats. To do so, we have generated 60000 symbols, subdivided equally between three time intervals where the applied modulation format is sequentially quadrature phase-shift keying (QPSK), 16-QAM and 64-QAM. The simulation was accomplished under Tx-IQ imbalances $\left(\epsilon_{p}=0.1, \phi_{p}=6^{\circ}\right)$ introduced on both $X$ and $Y$ polarizations, and polarization rotation parameters $\left(\alpha=30^{\circ}, \theta=50^{\circ}\right)$. The SNR is set to $23 \mathrm{~dB}$ for all the modulation formats. Also, the step-sizes are fixed in order to have the same initial convergence.

Figure 9 shows the MSE evolution for "CMA+BASS", "EASI+BASS" and the "joint" algorithms. "EASI+BASS" stands for EASI algorithm cascaded with the BASS. In Fig 9a, the radius of the CMA algorithm is kept to the value calculated for QPSK modulation. In this case, the CMA algorithm has no information about the change of the modulation format that is happening thereafter. Indeed, when the modulation format is changed at the transmitter from QPSK to 16-QAM, the CMA algorithm demonstrates a very slow coefficients adaptation compared with the "joint" algorithm. Moreover, the "CMA+BASS" algorithm maintains a slightly higher steady state convergence than the "joint" algorithm. In the contrary, EASI reacts well when the modulation format is changed at the transmitter from QPSK to 16-QAM but it maintains a slightly higher steady state convergence than the "joint" algorithm. 
Figure $9 \mathrm{~b}$ demonstrates the MSE trends when the CMA algorithm changes its radius according the simulated modulation format. This corresponds to the ideal situation when the CMA is optimally informed about the modulation format that is used in the transmitter. In this case, the "CMA+BASS" converges rapidly but still has a higher steady state performance than the "joint" algorithm. Performance of "EASI+BASS" and "CMA+BASS" are globally the same. However, in both cases, the "joint" algorithm reacts well regardless of the introduced modulation formats and maintains good performance in terms of speed of convergence and steady state values, unlike "CMA+BASS".

Before handling the complexity comparison part, we notice that the "joint" algorithm is not functional in the presence of strong DGD. Figure 10 depicts the effect of uncompensated residual delay on the performance of the "joint". Same numerical simulation parameters are maintained as before. The more the residual delay is, the more - MSE quantity (Mean Square Error of the obtained QAM signals) decreases. This issue is resolved by compensating the DGD in advance.

\section{COMPLEXITY COMPARISON}

In this section, the required resources of both "CMA+BASS" and the "joint" algorithm are compared

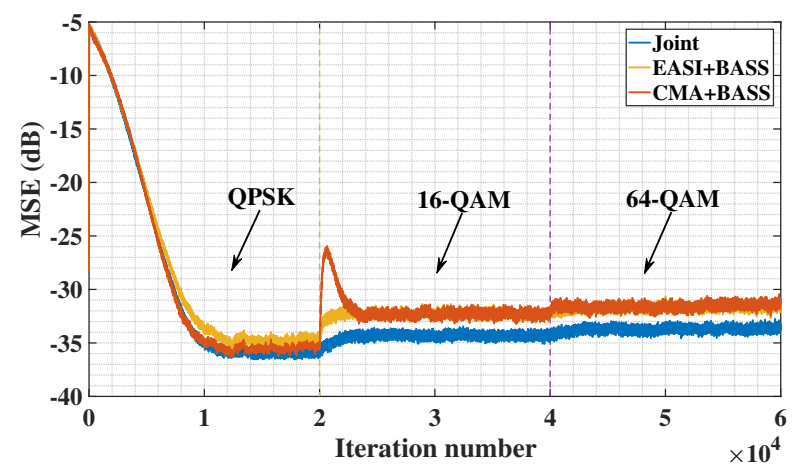

(a)

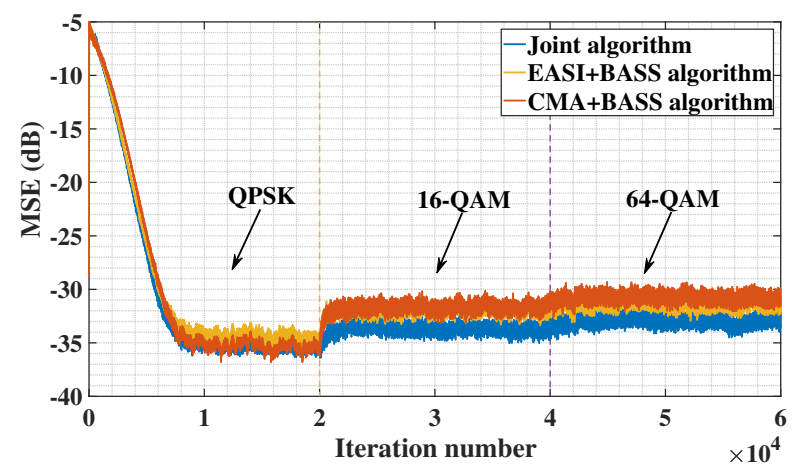

(b)

Figure 9: MSE curves in the presence of Tx-IQ imbalance and polarization rotation parameters for different modulation orders (QPSK,16-QAM and 64-QAM)(a) CMA is unaware of the change of modulation format, (b) CMA is made aware of the change of modulation format.

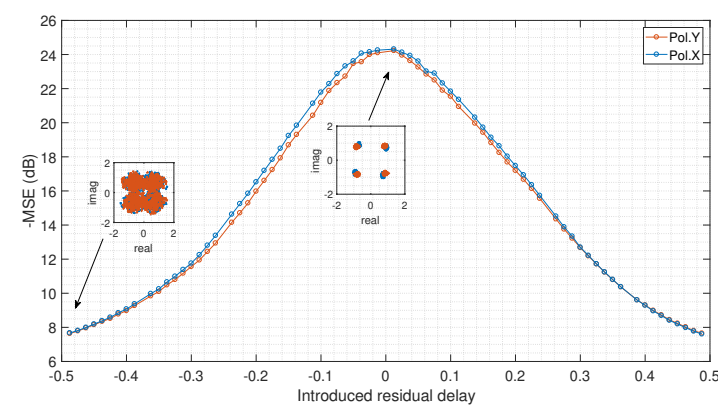

Figure 10: Effect of DGD on the $E s / N_{0}$

in the presence of residual chromatic dispersion. Regarding "CMA+BASS" algorithm, we evaluate its IQ imbalance compensator as well as its taps weight update complexity. As for the "joint" algorithm, it will be cascaded with two complex FIR filters to ensure residual chromatic dispersion compensation. Therefore, we will quantify both algorithms tap weight update computational load. In the above, we consider only the number of real multiplications and additions as they are assumed to dominate the computation cost of the update operations. Indeed, the multiplication operations are considered to be more computationally demanding than the addition. Table I presents a summary of the arithmetic needs of the "CMA+BASS" and the "joint" algorithms in terms of real multiplications and real additions per symbol. We have taken into account that a complex multiplication requires four real multiplications and two real additions.

Table I: Computational complexity of the proposed algorithm as compared with "CMA+BASS" algorithm

\begin{tabular}{|l|l|l|l|}
\cline { 2 - 4 } \multicolumn{1}{c|}{} & "CMA+BASS" & $\begin{array}{l}\text { "Joint+2 } \\
\text { FIR" }\end{array}$ & $\begin{array}{l}\text { "EASI+2 } \\
\text { FIR+BASS" }\end{array}$ \\
\hline Multiplications & $16 L+26$ & $\begin{array}{l}8 m^{2}+8 L+ \\
2\end{array}$ & $\begin{array}{l}8 m^{2}+8 L+ \\
24\end{array}$ \\
\hline Additions & $13+16 L$ & $6 m^{2}+8 L$ & $\begin{array}{l}6 m^{2}+8 L+ \\
13\end{array}$ \\
\hline $\begin{array}{l}\text { Multiplication } \\
\text { practical case }\end{array}$ & $16 L+26$ & $8 L+130$ & $8 L+152$ \\
\hline
\end{tabular}

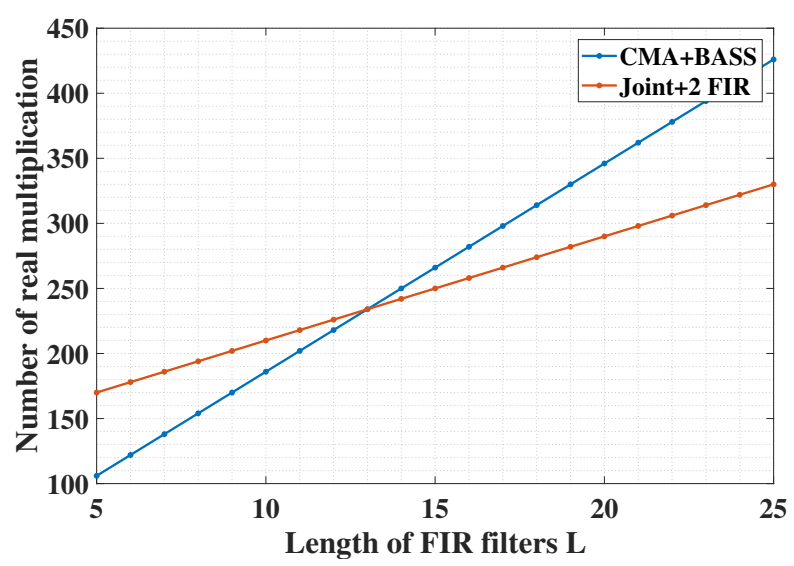

Figure 11: Number of real multiplications versus length of FIRs filters per sample.

$L$ is the length of the finite impulse response filters used 
in the CMA algorithm and $m$ is the number of sources to separate. Figure 11 presents the number of real multiplications of the two presented algorithms per sample. In our case, $m=4$. As can be seen in Fig 11, above $L=13$ taps, our proposed algorithm has less computational needs than the "CMA+BASS". This makes it a good tool for long-haul optical fiber transmission where CD impairments are a critical issue.

\section{CONCLUSION}

This article proposed a joint method for both Tx-IQ imbalance compensation and polarization demultiplexing in the absence of PMD and PDL. Even though residual CD persists, the presented method is still functional and requires one equalizer per polarization rather than four complex CMA filters. We studied by simulations the performance of our proposed algorithm in terms of MSE, EVM, BER metrics using PDM 16-QAM modulated signals. In term of MSE, it was shown that the proposed algorithm results in better steady state performance compared to the cascaded CMA and BASS algorithms. As for EVM and BER metrics, the two algorithms exhibit roughly the same performance. However, the salient point of our presented algorithm is its blind approach leading to fast speed of convergence regardless of the used modulation format, unlike its counterpart, which requires information about the used modulation to globally ensure the same performance. From a complexity point of view, the proposed algorithm remains less complex in terms of weight updating execution when compared to the "CMA+BASS" algorithm as it uses just two separate complex filters (i.e 4 filters with real taps). Finally, both proposed algorithms suffer from singularity as well as rotation ambiguities. However, singularity can be easily circumvented by inserting an appropriate constraint on the initial tap values, while rotation ambiguity is remedied by related DSP rotation block.

\section{REFERENCES}

[1] E. Ip, A. P. T. Lau, D. J. Barros, and J. M. Kahn, "Coherent detection in optical fiber systems," Opt. Express, vol. 16, no. 2, pp. 753-791, 2008.

[2] J. Renaudier et al., "Linear Fiber Impairments Mitigation of 40-Gbit/s Polarization-Multiplexed QPSK by Digital Processing in a Coherent Receiver," J. Lightw. Technol., vol. 26, no. 1, pp. 36-42, Jan. 2008.

[3] Y. Han and G. Li, "Coherent optical communication using polarization multiple-input-multiple-output," Opt. Express, vol. 13, no. 19, pp. 7527-7534, Sep. 2005.

[4] S. J. Savory, G. Gavioli, R. I. Killey, and P. Bayvel, "Transmission of $42.8 \mathrm{Gbit} / \mathrm{s}$ Polarization Multiplexed NRZ-QPSK over $6400 \mathrm{~km}$ of Standard Fiber with no Optical Dispersion Compensation," in Proc. Conference on Optical Fiber Communication and the National Fiber Optic Engineers Conference, Mar. 2007, pp. 1-3.

[5] A. Özen, I. Kaya, and B. Soysal, "A supervised constant modulus algorithm for blind equalization," Wireless Personal Commun., vol. 62, no. 1, pp. 151-166, jan. 2012.

[6] N. Stojanovic and X. Changsong, "An Efficient Method for Skew Estimation and Compensation in Coherent
Receivers," IEEE Photon. Technol. Lett., vol. 28, no. 4, pp. 489-492, Feb. 2016.

[7] T. Nguyen et al., "Blind transmitter IQ imbalance compensation in $M$-QaM optical coherent systems," IEEE/OSA J. Opt. Commun. and Netw., vol. 9, no. 9, pp. D42-D50, Sep. 2017.

[8] I. Fatadin, S. J. Savory, and D. Ives, "Compensation of Quadrature Imbalance in an Optical QPSK Coherent Receiver," IEEE Photon. Technol. Lett., vol. 20, no. 20, pp. 1733-1735, Oct. 2008.

[9] S. H. Chang, H. S. Chung, and K. Kim, "Impact of Quadrature Imbalance in Optical Coherent QPSK Receiver,' IEEE Photon. Technol. Lett., vol. 21, no. 11, pp. 709-711, Jun. 2009.

[10] C. S. Petrou, A. Vgenis, I. Roudas, and L. Raptis, "Quadrature Imbalance Compensation for PDM QPSK Coherent Optical Systems," IEEE Photon. Technol. Lett., vol. 21, no. 24, pp. 1876-1878, Dec. 2009.

[11] M. S. Faruk and K. Kikuchi, "Compensation for InPhase/Quadrature Imbalance in Coherent-Receiver Front End for Optical Quadrature Amplitude Modulation," IEEE Photon. J., vol. 5, no. 2, pp. 7800 110-7 800110 , Apr. 2013.

[12] T. Nguyen et al., "Joint simple blind IQ imbalance compensation and adaptive equalization for 16-QAM optical communications," in Proc. IEEE International Conference on Communications, Jun. 2015, pp. 49134918.

[13] M. Valkama, M. Renfors, and V. Koivunen, "Blind signal estimation in conjugate signal models with application to I/Q imbalance compensation," IEEE Signal Process. Lett., vol. 12, no. 11, pp. 733-736, Nov. 2005.

[14] J.-F. Cardoso and B. H. Laheld, "Equivariant adaptive source separation," IEEE Trans. Signal Process, vol. 44, no. 12, pp. 3017-3030, Dec. 1996.

[15] P. Rykaczewski, M. Valkama, and M. Renfors, "On the Connection of I/Q Imbalance and Channel Equalization in Direct-Conversion Transceivers," IEEE Trans. Veh. Technol., vol. 57, no. 3, pp. 1630-1636, May 2008.

[16] J. P. Gordon and H. Kogelnik, "PMD fundamentals: Polarization mode dispersion in optical fibers," Proc. Nat. Acad. of Sci., vol. 97, no. 9, pp. 4541-4550, 2000.

[17] H. Kogelnik, L. E. Nelson, and R. M. Jopson, "PMD characterization techniques," in Proc. IEEE Optical Fiber Communication Conference. Technical Digest Postconference Edition. Trends in Optics and Photonics Vol. 37, vol. 3, 2000, pp. 22-24.

[18] L. Liu et al., "Initial tap setup of constant modulus algorithm for polarization de-multiplexing in optical coherent receivers," in Proc. Conference on Optical Fiber Communication, Mar. 2009, pp. 1-3.

[19] S. Kun and Zhang Xudong, "A SE-CMA based blind equalization for MIMO systems," in Proc. IEEE International Conference on Signal Process., vol. 2, Aug. 2004, pp. 1674-1677. 